\title{
Comfortable Oscillation in Swinging Chair Using Heartbeat Fluctuation
}

\author{
Joichiro Eto ${ }^{\text {a }}$ Ryoichi Yagami ${ }^{\text {a }}$, Tatsuo Shimosako ${ }^{\text {a }}$, Takashi Soken ${ }^{\mathrm{a}}$, Keiji Matsumoto ${ }^{\mathrm{a}^{*}}$

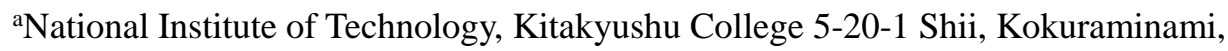 \\ Kitakyushu, Fukuoka, 802-0985, Japan \\ "Corresponding Author:kmatsu@kct.ac.jp
}

\begin{abstract}
In this study, a swinging chair that provides a comfortable swing is proposed. The objective of the proposed swinging chair is to generate oscillation using the ordinary swiveling chair driven by a small motor. The oscillation of the swinging chair is provided based on the signal generated using the fluctuations obtained from a person's heartbeat fluctuations. Instead of a standard questionnaire, the Index of Brain Resting State (IBRS), computed by the frequency transmitted from the brainwaves, is used to evaluate the comfort level.
\end{abstract}

Keywords: EEG, heartbeat, control, system.

\section{Introduction}

The evolution of technology has resulted in comfortable living, along with drastic changes in the social environment. However, the spread of computers has led to global competition among companies and cost reduction in employee's personnel. Consequently, people's stress levels are augmenting at an unprecedented rate. In recent years, research and development with respect to amenity and sensibility has been observed in various fields to address these problems [1-3]. The purpose of research on amenity is mainly to quantify the notion of sensory aspect. It requires insight beyond the realms of learning. The fields of research in regard to amenity are largely classified into four categories [4].
1. Living comfort
2. Ride quality
3. Sitting comfort of a chair
4. Clothing comfort

Living comfort is believed to be of foremost interest amongst these, for releasing daily stresses. In a typical living environment, the comfort is created through light, sound, and temperature, while the vibrational element is overlooked as a disagreeable element. However, the category of vibration includes both unpleasant and pleasant elements. An infant's cradle has wonderful features that provide comfort. See-saws, swings, and hammocks are practical examples of products that use swinging to release stress. Moreover, comfortable vibration in a car makes people lethargic. The active rocking chair has been developed that has employed this oscillation characteristic successfully [5-6]. In this study, the comfort of a rocking chair that is intrinsically controlled using a person's heartbeat fluctuation is evaluated using a questionnaire. The questionnaire evaluates parameters representing the psychological state of human beings on a numerical scale with five levels. However, the evaluated numerical value does not necessarily represent the psychological state as the humans' sense is relatively ambiguous.

In this study, a swinging chair that provides a comfortable swing is designed, and it is evaluated in terms of comfort using brainwaves. As mentioned above, although products utilizing the swinging characteristic exist, there is no product that provides a comfortable swing effortlessly by means of rotary motion. The swinging chair generates the oscillation using a regular swiveling chair driven by a motor. The oscillation of the swinging chair is provided based on the signal generated using the fluctuations obtained from the person's heartbeat fluctuations. Instead of a standard questionnaire, the Index of Brain Resting State (IBRS), computed by the frequency transmitted from the brainwaves, is used to evaluate the comfort level. It is believed that the system has the capability to relieve stress regardless of gender differences, age differences, or body type. 


\section{Method}

\subsection{Structure of the swinging chair}

A typical chair has either a fixed seat or a rotating seat. We believe that a comfortable chair can be realized by modifying a common chair. Fig. 1 shows the structure of the swinging chair. The swinging chair pleasantly oscillates, and its range of motion is limited to $180^{\circ}$. Fig. 2 shows the fabricated swinging chair and the structure of the rotating part. The rotating mechanism consists of a motor bracket, a pulley, and a timing belt. The driving motor is fixed on the bracket installed on the chair body. The pulley of diameter $120 \mathrm{~mm}$ is attached to the motor shaft. The axis rotating in synchronization with the chair seat has the same diameter as that of the pulley. The power from the motor is transmitted to the chair seat through the toothed belt that connects both the pulleys. A small DC motor of approximately $3 \mathrm{~W}$ is used to drive this mechanism. This motor is operated by the control box of the swinging chair. Fig. 3 shows the fabricated control box. The control box primarily consists of a heartbeat sensor, an Arduino, and a motor driver. The heartbeat waveform is transformed into the corresponding voltage using the heartbeat sensor attached to the human. This voltage power is transmitted to the Arduino. The Arduino computes the operation time of the swinging chair using the heartbeat fluctuations. The signal is transmitted from the Arduino to the power circuit board and amplified. Subsequently, the rotating motion is realized.

\subsection{Method for generating the signal containing the heartbeat fluctuation}

The average heart rate of a healthy adult is between 60 and 80, and the heartbeat period for such an adult is not a fixed cycle (Fig.4). The heartbeat fluctuation increases during the resting state and decreases under the stress state because the heart is influenced by both the sympathetic and parasympathetic nervous systems [7]. Therefore, it is possible to approximately comprehend the human state from the heartbeat fluctuations. In this study, the average heartbeat period of the human is believed to be the criterion, and the difference in each heartbeat period from the average value is the heartbeat fluctuation. Kawashima et al. reported the comfortable vibration frequencies through an experiment with a rocking chair operated at various frequencies using a motor [5, 6]. According to reports, a comfortable vibration is achieved when the amplitude changes with respect to the

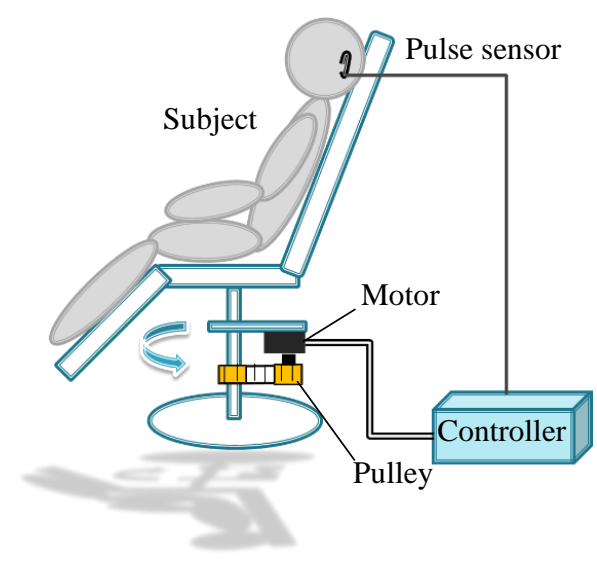

Fig.1 Structure of the swinging chair

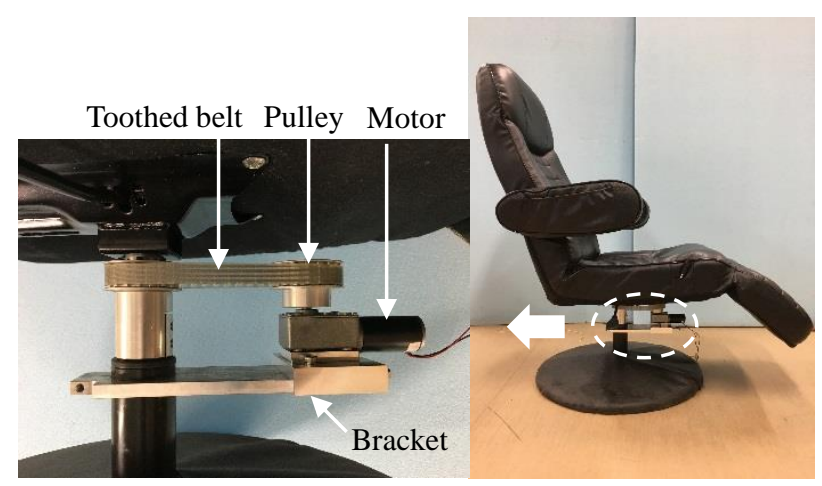

Fig.2 (1) rotating mechanism and (2) appearance of the swinging chair

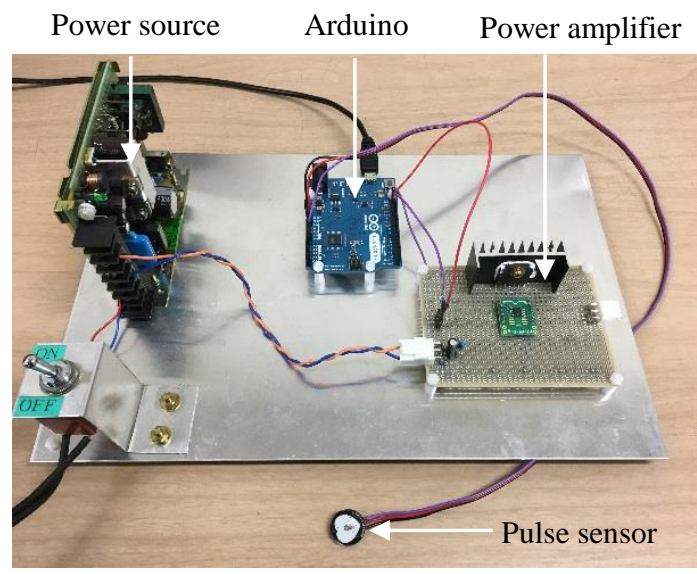

Fig.3 Fabricated control box for the swinging chair

criterion of 0.83 average heartbeat period. In this study, the heartbeat fluctuation value is assumed as the variation from the average heartbeat period during a minute. The swinging chair is operated by the motor control signal amplified by the heartbeat fluctuation value. The motor control signal is calculated using the following formula based on the driving 
algorithm proposed in the paper [5].

$$
T_{s 1}=1.446+1.847 \times \Delta T
$$

where the delta $\mathrm{T}$ is the average of five heartbeat fluctuations, and Ts1 is the rotating operation time. The swinging chair is operated by adding Ts1, acceleration time, and deceleration time to the motor control signal

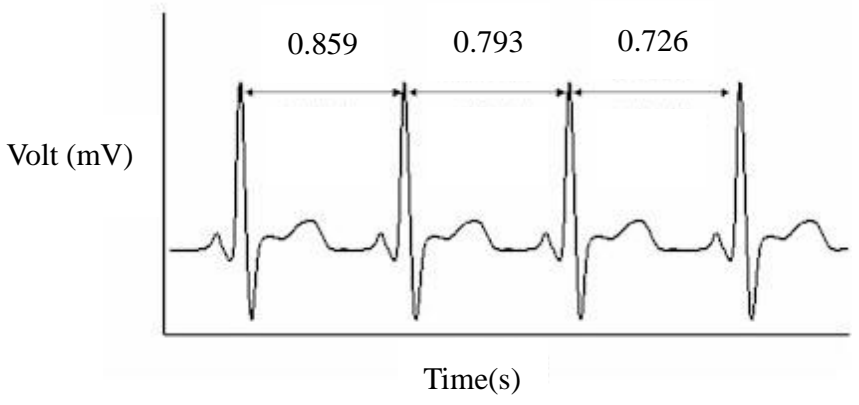

Fig.4 Heartbeat waveform in the time domain

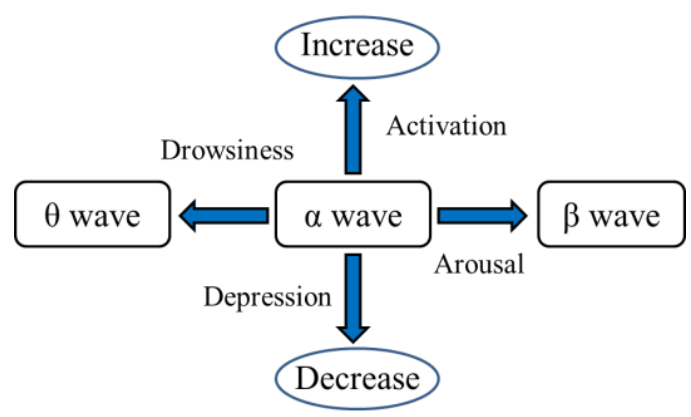

Fig.5 Relationship between the human state and brainwave frequencies

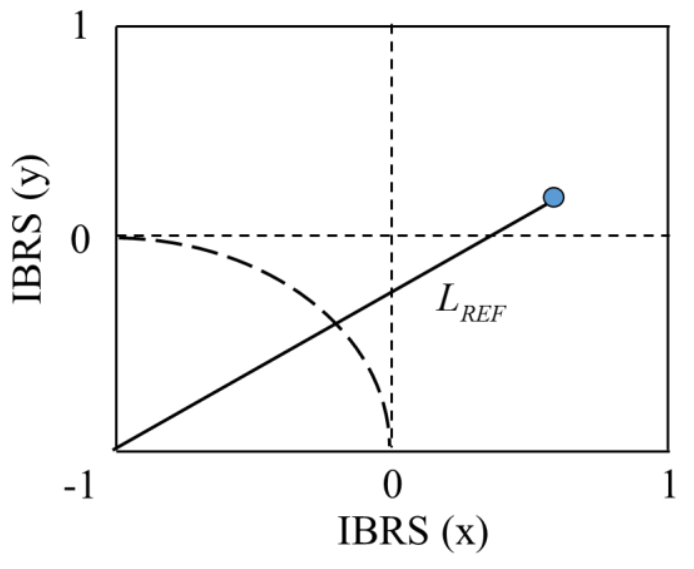

Fig.6 2D display of the IBRS

\subsection{Experimental procedure}

In order to evaluate comfort, a questionnaire followed by factor analysis using the semantic differential method is widely used [8]. The subject's impression is expressed by numerical of about 5 level for several items and obtained value is used as a psychological feature. However, the evaluated numerical value does not necessarily represent the psychological state. Thus, in this study, the psychological feature is evaluated using the brainwaves that are bio-information related to the human psychological state [9]. The subjects wearing the EEG sensor sit on the swinging chair and relax. Once the measurement begins, the swinging chair begins oscillating. The measurement is performed for $5 \mathrm{~min}$. The brainwave is detected by the EEG sensor and transmitted to a desktop computer for analysis during the measurement. The heartbeat measured by the heartbeat sensor is received at the Arduino. The subject's state is evaluated by means of the IBRS calculated using the measured brainwaves once the experiment is completed.

\subsection{EEG device}

In this study, the brainwaves are detected to estimate the human state using an EEG sensor. The single-channel EEG sensor manufactured by NeuroSky Inc. has two electrodes to measure brain potential: an active electrode and a reference electrode. The test subject wears the EEG sensor on their head, and it monitors the electrical potential between the electrodes attached to the forehead and earlobe. It is possible to analyze each frequency elements in detail, because the EEG signals are presented at every $0.25 \mathrm{~Hz}$ in the range of 0 to 255 frequency band [10]. All of the EEG signals were transmitted to a computer via a Bluetooth wireless communication device. These frequency domain signals are used for estimating the subject's state.

\section{$2.5 \quad$ IBRS}

IBSR is an evaluation method for the resting state of human based on an electroencephalographic index [11]. Figure. 5 shows the conceptual diagram of the human state and brainwave frequencies. Generally, the alpha wave is used as an index of resting, and its integral amplitude value is ordinarily used as the discrimination criterion. However, other brainwave information is also needed to correctly estimate the human state, because alpha waves are 
considerably affected by visual information. Generally, the beta-wave element increases, along with its frequency, if the brain enters an excited state. In contrast, when the brain becomes drowsy and enters a suppressed state, the thetawave element increases, and its frequency decreases. The condition between arousal and drowsiness is considered to be the brain-resting state. Therefore, the amplitude of the alpha wave compared to those of the theta and beta waves is defined as the IBRS. Formulas for the IBRS are defined as follows.

$$
\begin{gathered}
X_{I B R S}=\frac{\theta_{s t r}-\alpha_{s t r}}{\theta_{s t r}+\alpha_{s t r}} \\
Y_{I B R S}=\frac{\beta_{s t r}-\alpha_{s t r}}{\beta_{s t r}+\alpha_{s t r}}
\end{gathered}
$$

The IBRS is visually indicated in two dimensions using the ratio between the theta and alpha waves or between the beta and alpha waves (Fig.6). The horizontal axis is defined as the IBRS' $\mathrm{X}$ component, which is represented by both the theta and alpha waves, and the vertical axis is defined as the IBRS' $\mathrm{Y}$ component, which is represented by the beta and alpha waves. Both components of the IBRS are represented in a range of -1 to +1 . The value gradually becomes closer to -1 as the alpha wave increases. When the point $(-1,-1)$ is assumed to be the origin, the resting rate increases when the point indicated as the IBRS coordinate moves closer to the origin. The vector length from the origin is defined as the IBRS, as shown in Fig.6. We estimated the human state using this index.

\section{Result and discussion}

Initially, the experiment was carried out with an ordinary chair to compare with the comfort of the swinging chair. Fig.7 shows the IBRS obtained by calculating the measured EEG. It is observed that the IBRS after approximately $160 \mathrm{~s}$ was mostly less than 1.0 . The average value of the IBRS from 0 to $160 \mathrm{~s}$ was 1.23 , and the average from 160 to $300 \mathrm{~s}$ was 0.85 . It was confirmed that, in the interval between 160 and $300 \mathrm{~s}$, alpha waves were predominant over theta and beta waves. The subject seemed to relax as the experiment progressed. Fig.8 shows the transition of the heartbeat period with respect to time. Here, the same period of the IBRS as mentioned above was considered. The average heartbeat period in the interval between 0 and $160 \mathrm{~s}$ was $995.3 \mathrm{~ms}$ and the same between 160 and $300 \mathrm{~s}$ was $984.1 \mathrm{~ms}$. The standard deviation was 57.3 in the interval from 0 to $160 \mathrm{~s}$, and 106.7 in the interval from 160 to $300 \mathrm{~s}$. It was confirmed that the variation in the heartbeat period in the interval from 160 to $300 \mathrm{~s}$ was relatively large, because there was a difference of 49.36 in the standard deviation. It implies that the subject was relaxed during the period.

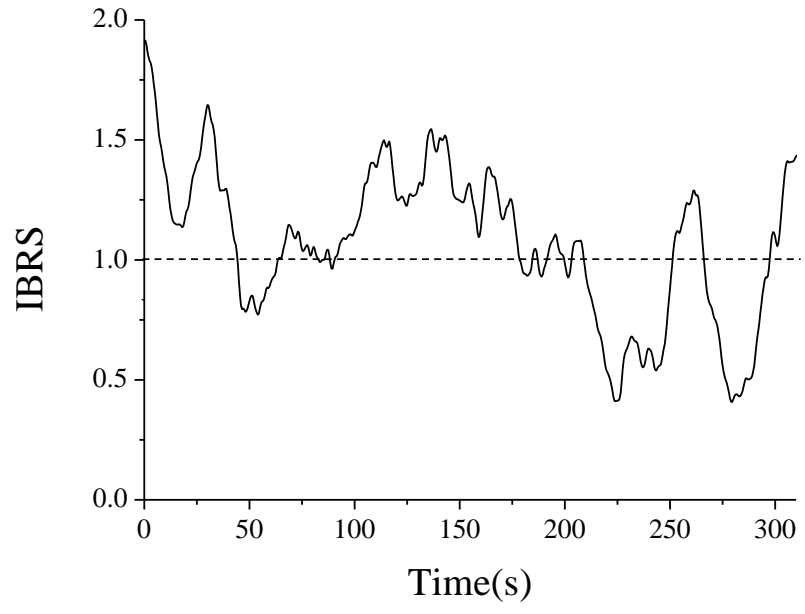

Fig.7 The time transition of IBRS using ordinary chair

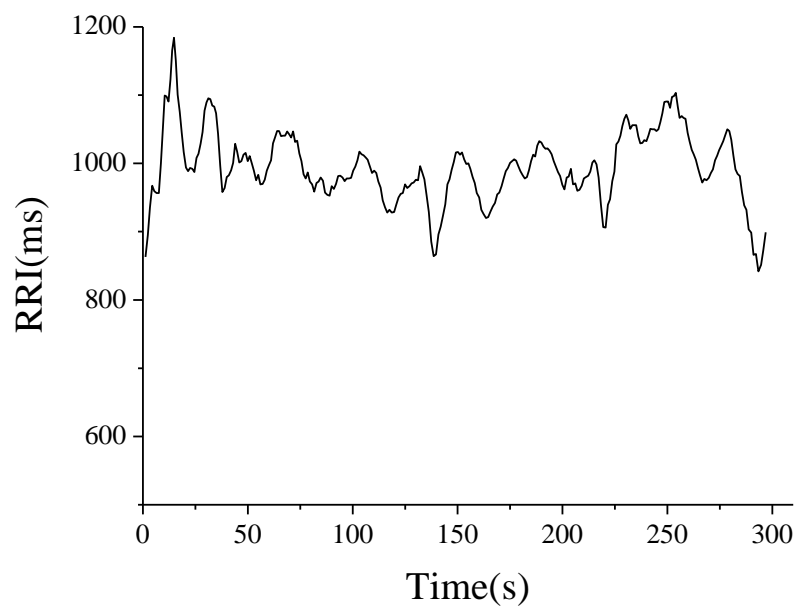

Fig.8 The time transition of heartbeat period using ordinary chair

The same experiment was carried out using the swinging chair. Fig.9 shows the IBRS calculated from the measured value, similar to the case with the ordinary chair. The IBRS was equal to or less than 1.0 after approximately $25 \mathrm{~s}$. Thereafter, it showed 1.0 or less until the end of the measurement. The average value from 25 to $300 \mathrm{~s}$ was 0.72 , which is less than that of the ordinary chair. This implies that the subject was relaxed and that the relative dominance of the alpha waves was larger for the swinging chair when compared with the ordinary chair. Fig.10 shows the transition of heartbeat period in the time domain for the swinging chair. The average heartbeat period and standard deviation in the interval from 25 to $300 \mathrm{~s}$ were $843.3 \mathrm{~ms}$ and 
98.9, respectively. Owing to the large fluctuation in the heartbeat, it can be concluded that the subject was relaxed. We observed the following when comparing the two experimental results. The swinging chair reduced the time taken by the subject to relax from $160 \mathrm{~s}$ to $25 \mathrm{~s}$. This implies that the swinging chair created a comfortable condition much sooner. Since the IBRS during the relaxing state was 0.85 and 0.72 , respectively for the two chairs, we inferred that the alpha wave in case of the swinging chair was larger than that of the ordinary chair. Moreover, in case of the ordinary chair, the variation in the IBRS was relatively high, whereas the IBRS in case of the swinging chair was stable at 1.0 or less. It was concluded that the swinging chair created a stable comfortable condition for a long period of time when compared with an ordinary chair. From the above results, we can validate the effectiveness of the swinging chair in providing comfort and relaxation to people.

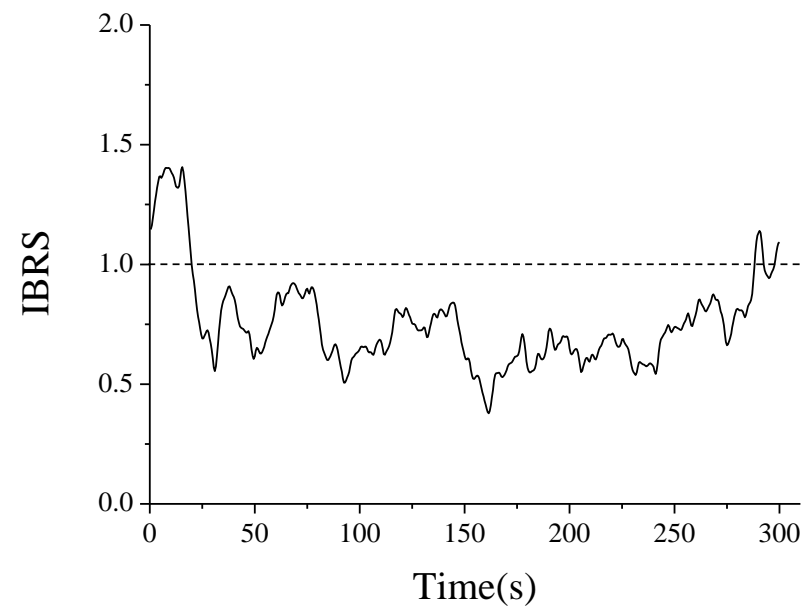

Fig.9 The time transition of IBRS using swinging chair

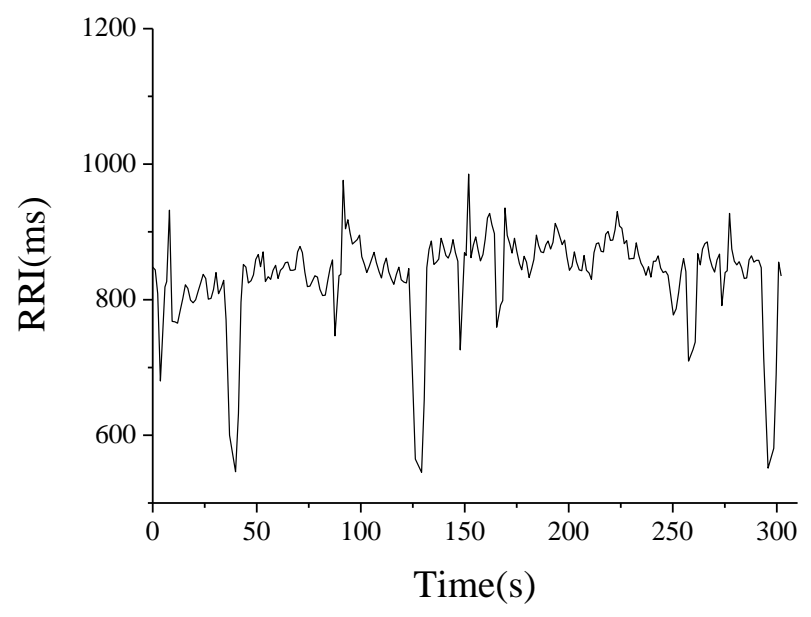

Fig.10 The time transition of RRI using swinging chair

\section{Conclusion}

In this study, a swinging chair that provides a comfortable swing using a person's heartbeat was designed and fabricated. Its effectiveness in providing comfort was evaluated using heartbeat and brainwaves. An ordinary chair installed with a rotating mechanism was employed to realize the swinging chair. An experiment using a normal chair and swinging chair was carried out in order to evaluate the comfort of a fabricated swinging chair. The average value of the IBRS in the interval between 0 and $160 \mathrm{~s}$ was 1.23 , and the same value in the interval between 160 and $300 \mathrm{~s}$ was 0.85 . The standard deviation was 57.3 in the interval from 0 to $160 \mathrm{~s}$, and 106.7 in the section from 160 to $300 \mathrm{~s}$. It was confirmed that the variation in heartbeat period in the section from 160 to $300 \mathrm{~s}$ was relatively large, because there was a difference of 49.36 in standard deviation. The subject seemed to be relaxed during the interval between 160 and $300 \mathrm{~s}$, because the variation in the heartbeat period in this interval was relatively large and the IBRS was less than 1.0. In case of the swinging chair, the IBRS was equal to or less than 1.0 after approximately $25 \mathrm{~s}$. Thereafter it was 1.0 or less until the end of measurement. The average value of the IBRS and the standard deviation of the heartbeat period in the interval between 25 and $300 \mathrm{~s}$ were 0.72 and 98.9, respectively. These results show that the subject was in a relaxed state after approximately $25 \mathrm{~s}$ and the state continued until the end of the measurement. From the above results, we can confirm the effectiveness of the swinging chair in providing comfort and relaxation to people.

\section{References}

(1) T.Kotani, H.Higashi, "Approach for Realization of Comfortable Lighting Environment Using LED Luminaires", Toshiba review, Vol.65, No.7, pp.20-23, 2010.

(2) B.Ko, D.Lee, H.Chiang, T.Koga, and K.Hirate, "Basic Study on Rest Effect of Light Color in the LED Lighting”, J. Environ. Eng. AIJ, Vol.76, No.662, pp.363368, 2011.

(3) F.Wang, K.Sagawa \& H.Inooka, "A study of the relationship between the longitudinal acceleration/deceleration of automobiles and ride comfort", human engineering, Vol.36, No.4, pp.191-200, 2000 . 
(4) K.Suzuki, "Measure comfort: evaluation of its psychological, behavioral and physiological effects, Japan Publication Service, 2002, in Japanese.

(5) T.Kawashima, "Study for Comfortable Swinging: Comfortable Swinging for Active Rocking Chair", The Japan Society of Mechanical Engineers, Vol.69, No.677, pp.219-226, 2003.

(6) T.Kawashima, "Development of Active Rocking Chair Changing the Swing with Heartbeat Fluctuation", The Japan Society of Mechanical Engineers, Vol.71, No.709, pp.2731-2737, 2005.

(7) J.Hayano, "Biosignal monitoring during daily life: What we will see through new windows", Nagoya Med. J, Vol.59, pp.93-99, 2009.
(8) M.Nagamachi, "Kansei engineering: technology of human senses in design (In Japanese)", Kaibundo Publishers, 1989.

(9) T.Ichikawa, “An Invitation to Journey for Understanding of EEG”, Seiwa Shoten Publishers, 2006, in Japanese.

(10) NeuroSky, "MindSet Communications Protocol", NeuroSky application note (2011).

(11) Kaoru Yoshii and Keiji Matsumoto, "A Dry-Eye Warning System Based on the Estimation of the Human State Using a Single-Channel EEG Sensor", Proceedings of the 4th IIAE International Conference on Intelligent Systems and Image Processing 2016, pp.142148, Sep, 2016 